\title{
Capacity Analysis of OFDM / FBMC based Cognitive Radio Networks with Estimated CSI
}

\author{
Haijian ZHANG* ${ }^{* \dagger}$, Didier LE RUYET*, Daniel ROVIRAS*, and Hong SUN ${ }^{\dagger}$ \\ *Electronics and Communications Laboratory, CNAM, Paris, France \\ ${ }^{\dagger}$ Signal Processing Laboratory, Wuhan University, China \\ haijian.zhang@cnam.fr, leruyet@cnam.fr, daniel.roviras@cnam.fr, hongsun@whu.edu.cn
}

\begin{abstract}
In this paper, we focus on the channel capacity analysis of a Cognitive Radio (CR) network using two types of multicarrier communications: conventional Orthogonal Frequency Division Multiplexing (OFDM) with Cyclic Prefix (CP) and Filter Bank based Multi-Carrier (FBMC) modulations. We use a resource allocation algorithm in which subcarrier assignment and power allocation are carried out sequentially. By taking the impact of Inter-Cell Interference (ICI) resulting from timing offset into account, the maximization of total information rates is formulated, subject to maximum power constraint as well as interference constraint from Secondary User (SU) to Primary User (PU). Since the channel gain information between $\mathrm{SU}$ and PU can't be precisely estimated, the amount of the interference induced by $\mathrm{SU}$ to $\mathrm{PU}$ is calculated on the basis of an estimated Channel State Information (CSI), which is determined by a prescribed outage probability of primary systems. Simulation results show that FBMC can achieve higher channel capacity than OFDM, which indicates that FBMC is a potential candidate for physical layer data communication in CR context. ${ }^{1}$
\end{abstract}

\section{INTRODUCTION}

Much of attention in the Cognitive Radio (CR) literature emphasizes on the use of conventional Orthogonal Frequency Division Multiplexing (OFDM). However, OFDM is very sensitive to fast time variations of the radio channel and to timing offset due to imperfect synchronization. The shortcoming for OFDM based systems originates from the large side-lobes of the frequency response of the filters. These side-lobes, in turn, result in significant interference among subcarriers.

The Filter Bank based Multi-Carrier (FBMC) is another alternative multicarrier modulation [1][2], which does not require $\mathrm{CP}$ extension and shows higher robustness to residual frequency offsets than CP-OFDM by taking advantage of the low side-lobe of its modulation prototype filters. In [3], the author proposed the utilization of a FBMC technique, termed Offset Quadrature Amplitude Modulation (OQAM) to ease the leakage problem of OFDM. In [4], FBMC communication technique is verified to offer higher spectral efficiency than OFDM in the context of CR. Moreover, filter banks can be used as a tool for spectrum sensing. In [5], application of filter banks to spectrum sensing is proved to be more promising than Fast Fourier Transform (FFT) components and Thomson's Multi-Taper (MT) method.

\footnotetext{
${ }^{1}$ This work was supported in part by the European Commission under Project PHYDYAS (FP7-ICT-2007-1-211887)
}

Optimal resource allocation problem in multicarrier CR context with both power and mutual interference constraints is still an open topic. In [6], a computationally efficient resource allocation algorithm in multicarrier based CR systems is proposed. However, the interference induced from Primary User (PU) to Secondary User (SU) is assumed to be negligible and channel pathloss is not considered. In [7], the authors propose a resource allocation scheme under an uplink scenario with pathloss and Rayleigh channel, and a maximization of sum-rate is formulated with both power constraint and inter-cell interference [8] constraint. In order to compute the amount of interference form SU to PU, the channel gain information between SU and PU is assumed to be known by SU. Nevertheless, under the hypothesis that primary and secondary systems are unsynchronized, it's hard to perfectly estimate this channel gain.

This paper is an extension based on our previous work in [7]. We focus on the comparison of OFDM and FBMC based CR networks in terms of the averaged channel capacity, which depends on the resource allocation strategy adopted by the CR system. We use the resource allocation scheme defined in [7], which is split into two steps to reduce the complexity. First of all, SUs are assigned to the detected spectrum holes, which is implemented by using a proposed Averaged Capacity metric (AC-metric). After assigning SUs to spectrum holes, the power allocation is solved by the Gradient Projection Method (GPM) [9] instead of using the complex Lagrangian multiplier method. Standards for multicarriers CR systems are still under study and no common definition for interference threshold is available in the literature. Herein we define this interference threshold by using a tolerable capacity loss for primary users. The first difference from [7] is that we consider the capacity loss on all the subcarriers that one PU occupies instead of on the first primary subcarrier adjacent to SU. Secondly, we assume that a rough estimate of the channel gain from SU to PU can be obtained by the SU during the spectrum sensing phase. The estimation error is determined by a prescribed outage probability of primary systems. Based on a rough estimated channel gain, final simulation results show more distinct performance difference between FBMC and OFDM than the case with an ideal channel gain estimation.

In the following, we present the system model and formulate our problem in Section II. In Section III, the proposed resource 
allocation algorithm for multi-user is introduced. Simulation results are given in Section IV. Finally, conclusion is drawn in Section V.

\section{SYSTEM MODEL AND PROBLEM FORMULATION}

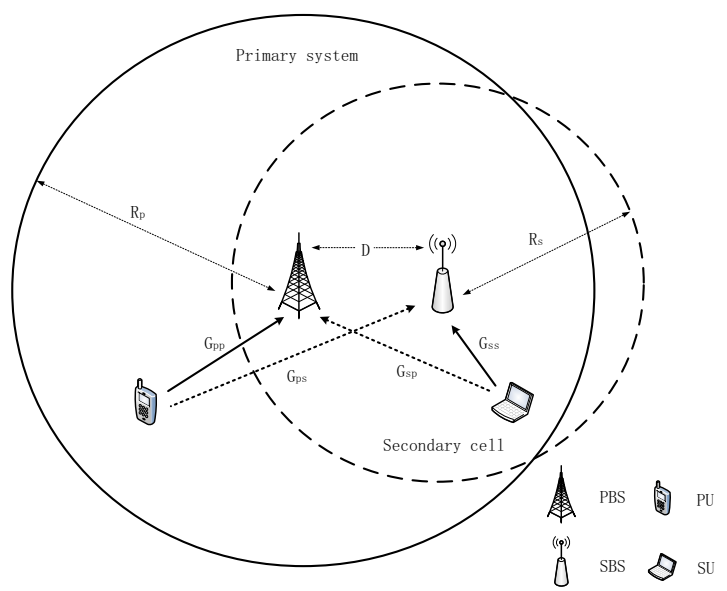

Fig. 1. CR networks with one primary system and one secondary cell

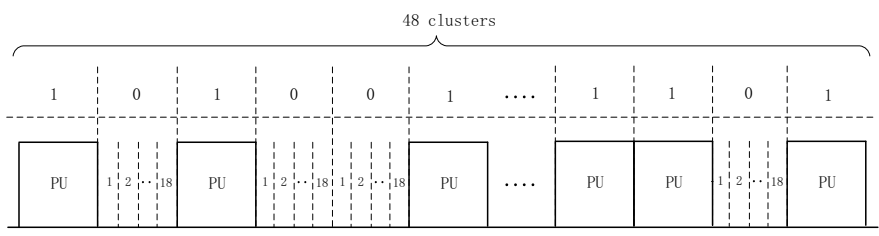

Fig. 2. Distributions of the primary users and the spectrum holes with $N_{\text {all }}=48$ and $L=18$

As shown in Fig. 1, an uplink scenario of CR networks consisting of one primary system with one PU and one secondary cell with one $\mathrm{SU}$ is graphed, where $D$ is the distance between the Primary Base Station (PBS) and Secondary Base Station (SBS), and $R_{p}$ and $R_{s}$ are the radius of primary system and secondary cell, respectively. A frequency band of $N_{\text {all }}$ clusters with $L$ subcarriers in each cluster is licensed to primary system. Fig. 2 shows the distributions of the primary users (referred to as "1") and the spectrum holes (referred to as "0") with $N_{\text {all }}=48$ and $L=18 .{ }^{2}$

Given above basic uplink scenario, we make the following assumptions for our system model:

1) Primary system and secondary cell apply the same multicarrier modulation scheme (OFDM or FBMC).

2) SUs in the secondary cell are synchronized, and SBS can perfectly sense the free bands of the licensed system.

3) SBS has the channel gain knowledge of $G_{s s}$ (indicated in Fig. 1).

4) Primary system and secondary cell are assumed to be unsynchronized, so ICI exists between PU and SU.

\footnotetext{
${ }^{2}$ Here we have chosen the practical values of WIMAX 802.16 for the number and size of clusters.
}

5) We consider a flat fading Rayleigh channel, and we assume that the channel changes slowly so that the channel gains will be constant during transmission.

In [8], averaged OFDM/FBMC ICI tables have been obtained when transmitting a single complex symbol with power that equals to " 1 " on the $k^{\text {th }}$ frequency slot and the $n^{\text {th }}$ time slot. As shown in Fig. 2, the primary users and secondary users share adjacent frequency bands, and one spectrum hole might have one or multiple clusters, that is, one secondary user is permitted to occupy at least $L$ subcarriers. Nevertheless, according to the obtained OFDM/FBMC ICI tables in [8], only "1" subcarrier (FBMC) or " 8 " subcarriers (OFDM) really induces ICI to primary users.

When we transmit a burst of independent complex symbols, the interference incurred by one subcarrier equals to the sum of the interference for all the time slots. For our following theoretical analysis, the eight-element interference vectors of OFDM with CP length of $T / 8$ ( $\mathrm{T}$ indicates one symbol period) and FBMC designed in [2] are defined as

$$
\begin{aligned}
& V^{o f d m}=10^{-2} \times[8.94, \quad 2.23, \quad 0.995, \quad 0.560, \\
& 0.359, \quad 0.250, \quad 0.184, \quad 0.112] \\
& V^{f b m c}=10^{-2} \times\left[\begin{array}{llllllll}
8.81, & 0, & 0, & 0, & 0, & 0, & 0, & 0
\end{array}\right]
\end{aligned}
$$

The secondary cell wants to maximize its sum data rate by allocating power into the detected spectrum holes for its own users, this problem can be formulated as

$$
\max _{\mathbf{p}}: C(\mathbf{p})=\sum_{m=1}^{M} \sum_{k=1}^{K} \sum_{f=1}^{F_{k}} \theta_{m}^{k f} \log _{2}\left[1+\frac{p_{m}^{k f} G_{s s}^{m k f}}{\sigma^{2}+I_{f}^{k}}\right]
$$

s.t.

$$
\begin{aligned}
& \sum_{k=1}^{K} \sum_{f=1}^{F_{k}} \theta_{m}^{k f} p_{m}^{k f} \leq P_{t h}, \quad \forall m ; \\
& 0 \leq p_{m}^{k f} \leq P_{s u b} ; \\
& \sum_{m=1}^{M} \sum_{n=1}^{N} \theta_{m}^{k_{l(r)} n} p_{m}^{k_{l(r)} n} G_{s p}^{m k_{l(r)}} \sum_{i=1}^{N-n+1} V_{N-i+1} \leq I_{t h}, \forall k ;
\end{aligned}
$$

where $M$ is the number of secondary users, $K$ is the number of spectrum holes, and $F_{k}$ is the number of subcarriers in the $k^{t h}$ spectrum hole. $\theta_{m}^{k f} \in\{0,1\}$ is the subcarrier assignment indicator, i.e. $\theta_{m}^{k f}=1$ if the $f^{t h}$ subcarrier in the $k^{t h}$ spectrum hole is allocated to SU $m, p_{m}^{k f}$ is the power of SU $m$ on the $f^{t h}$ subcarrier in the $k^{t h}$ spectrum hole, $G_{s s}^{m k f}$ is the propagation channel gain from SU $m$ to SBS on the $f^{t h}$ subcarrier in the $k^{t h}$ spectrum hole, $\sigma^{2}$ is the noise power, and $I_{f}^{k}$ is the ICI from PU to $\mathrm{SU}$ on the $f^{\text {th }}$ subcarrier in the $k^{\text {th }}$ spectrum hole. $P_{t h}$ and $P_{s u b}$ are the maximum user power limit and per subcarrier power limit, respectively. $N$ is the length of the interference vector $V, p_{m}^{k_{l(r)} n}$ is the power of SU $m$ on the left (right) $n^{t h}$ subcarrier in the $k^{t h}$ spectrum hole, $G_{s p}^{m k_{l(r)}}$ is the propagation channel gain from SU $m$ to PBS on the left 
(right) primary subcarriers adjacent to the $k^{t h}$ spectrum hole, and $I_{t h}$ denotes the interference threshold.

The ICI from PU to SU $I_{f}^{k}$ can be expressed in the mathematical form as follows

$$
I_{f}^{k}=\left\{\begin{array}{cc}
\sum_{n=f}^{N} P_{p}^{k_{l}} G_{p s}^{k_{l} f} V_{n}, \quad f=1,2, \cdots N \\
\sum_{n=F_{k}-f+1}^{N} P_{p}^{k_{r}} G_{p s}^{k_{r} f} V_{n}, f=F_{k}-N+1, \cdots F_{k} \\
0, & \text { otherwise }
\end{array}\right.
$$

where $P_{p}^{k_{l(r)}}$ is the transmission power of PU located in the left (right) of the $k^{t h}$ spectrum hole, and $G_{p s}^{k_{l(r)} f}$ is the channel gain from PU located in the left (right) of the $k^{t h}$ spectrum hole to SBS on the $f^{t h}$ subcarrier of the $k^{t h}$ spectrum hole. Generally, the secondary cell is not capable of obtaining the transmission power of PU and the channel information from PU to SU, but $I_{f}^{k}$ can be measured during the spectrum sensing by SBS without need to know these information.

The third inequality constraint in (2) is related to the interference introduced by $\mathrm{SU}$ on its adjacent $N$ primary subcarriers. This constraint is quite difficult to manage due to: first of all, the threshold $I_{t h}$ has to be prescribed by the primary system. Different thresholds corresponding to different penalties in terms of primary system capacity degradation will be defined and used in section IV of this paper. Secondly, the SU needs the necessary channel knowledge. Without the information of the channel gain $G_{s p}$ between SU and PBS, the third term of the inequality constraint of (2) can't be computed. Practically, it's hard to perfectly estimate $G_{s p}$. Nevertheless, the pathloss gain $G_{p l}$ from PBS to SU can be estimated on the subcarriers used by primary system and, by interpolation, the channel gain $G_{s p}$ on free subcarriers can be computed. The channel gain of the downlink path is not equal to the reverse channel gain if Frequency Division Duplexing (FDD) is used. In this case the downlink channel gain can be used as a rough estimate of the uplink channel gain.

In order to guarantee the Quality of Service (QoS) of primary systems, a channel gain margin $G_{m}$ is added on the estimated pathloss gain

$$
\overline{G_{s p}}=\left(1+G_{m}\right) G_{p l}
$$

where $G_{m}$ depends on the prescribed outage probability $P_{\text {out }}$ tolerated by primary systems. Based on the implicit assumption that downlink and uplink pathloss difference is negligible, the evaluation of $\overline{G_{s p}}$ depends only on the Rayleigh fading. We define the outage probability as

$$
P_{\text {out }}=P\left(G_{s p}>\overline{G_{s p}}\right)=P\left(\left|H_{s p}\right|^{2}>1+G_{m}\right)
$$

where $G_{s p}=\left|H_{s p}\right|^{2} G_{p l}, H_{s p}$ is the Rayleigh fading frequency response. Since $H_{s p} \sim \operatorname{Rayleigh}(\mu)$, then $\left|H_{s p}\right|^{2}$ has a Gamma distribution with shape parameter $\alpha=1$ and scale parameter $\beta=2 \mu^{2}$. The cumulative distribution function (CDF) of $\left|H_{s p}\right|^{2}$ is the regularized gamma function, therefore (5) can be further expressed as

$$
1-P_{\text {out }}=\frac{\gamma\left(\alpha,\left(1+G_{m}\right) / \beta\right)}{\Gamma(\alpha)}
$$

where $\gamma$ is the lower incomplete gamma function. Given an acceptable outage probability $P_{\text {out }}$, the added channel gain margin $G_{m}$ can be obtained by (6)

$$
G_{m}=2 \mu^{2} \log _{e}\left(\frac{1}{P_{\text {out }}}\right)-1
$$

\section{MULTI-USER RESOURCE ALLOCATION}

In multicarrier based networks with multi-user, assuming each free subcarrier can be used for transmission by at most one secondary user at any time, the optimal problem in (2) is an integer programming problem, which has a high computational complexity. Generally, instead of searching an optimal solution with an unacceptable computational complexity, the combinatorial suboptimal method of subcarrier assignment and power allocation is proposed: firstly the subcarriers are assigned to the SUs and then the power is allocated to these subcarriers.

\section{A. Cluster Assignment}

The first task of subcarrier assignment for our multi-user scenario is the bandwidth allocation. In order to guarantee the fairness for our rate adaptive optimization problem, the bandwidth allocation method in [10] is applied to assign the number of clusters to each SU. The number of clusters $N_{u}$ for user $u$ is determined by providing a degree of fairness. The bandwidth assignment algorithm increments $N_{u}$ for the user $u$ which exhibits the smallest channel capacity, and the algorithm ends when the total number of clusters assigned to SUs equals to the number of free clusters.

Afterwards the specific subcarrier assignment is examined. The Averaged Capacity metric (AC-metric) aiming to maximize the averaged channel capacity proposed in [7] is applied for the cluster assignment. The averaged channel capacity depends not only on the channel gain $G_{s s}$, but also on the interference threshold $I_{t h}$, maximum user power limit $P_{t h}$, as well as the channel gain $G_{s p}$. AC-metric makes a balance between all these influence factors.

Assuming there are $K^{c}$ free clusters and $K^{u}$ secondary users, AC-metric can be used for calculating the averaged capacities of each SU on each available cluster. Since we know the number of clusters assigned to each secondary user using the bandwidth allocation method, a $K^{c} \times K^{c}$ AC matrix can be obtained. Our task is how to optimally assign these $K^{c}$ clusters to the $K^{u}$ secondary users, with the aim of maximizing the averaged spectral efficiency of the secondary cell. This problem equals to the search of the optimum matching of a bipartite graph, so the Hungarian algorithm introduced by $\mathrm{H}$. W. Kuhn [11] is proposed to implement this cluster assignment.

Mathematically, the cluster assignment problem can be described as: Given the $K^{c} \times K^{c}$ AC cost matrix $\mathcal{R}=\left[r_{m, n}\right]$, find the $K^{c} \times K^{c}$ permutation matrix $\Psi=\left[\psi_{m, n}\right]$ so that

$$
\mathcal{V}_{\psi}=\sum_{m=1}^{K^{c}} \sum_{n=1}^{K^{c}} \psi_{m, n} r_{m, n}
$$

is maximized. 
For the low dimension AC matrix, the optimal permutation matrix $\Psi$ can be obtained efficiently by using Hungarian algorithm.

\section{B. Power Allocation}

At the premise of knowing the result of the cluster assignment, the power allocation of multi-user system can be virtually regarded as a single-user system. For the special case of single-user, the problem formulation in (2) is simplified, where the $\mathrm{SU}$ is permitted to access all the $F=\sum_{k=1}^{K} F_{k}$ subcarriers.

In mathematical optimization, the method of Lagrangian multipliers can provide a strategy for finding the optimal solution, but the solution of extensive Lagrangian multipliers is computationally complex when $F$ increases. Herein Rosen's Gradient Projection Method (GPM) [9] can be applied to obtain the optimal power allocation for this simple CR uplink scenario with a low computational complexity.

\section{SIMULATIONS}

In this section, the proposed resource allocation algorithm of OFDM and FBMC based CR networks is evaluated in terms of the averaged channel capacity by computer simulations in a comparable way.

The CR network as shown in Fig. 1 with one primary system and one secondary cell is simulated. Without loss of generality for the proposed resource allocation algorithm, the case with multiple PUs and multiple SUs is considered. Assuming 36 clusters (seventy-five percent of the total $N_{\text {all }}=48$ clusters) are allocated to PUs, and these 36 licensed clusters are occupied by 36 uniformly distributed PUs. The rest 12 clusters are permitted to access by 6 SUs. Primary and secondary users centering around PBS and SBS, respectively, are uniformly distributed within the cell range $(0.1 \sim 1 \mathrm{~km})$. As the increase of transmission distance, the attenuation also increases due to the propagation pathloss. The pathloss of the received signal at a distance $d(\mathrm{~km})$ is [12]

$$
P(d)=128.1+37.6 \log _{10}(d) \quad d B
$$

Other system simulation parameters are displayed in Table I.

In order to define an interference threshold $I_{t h}$ which is predetermined by a practical licensed system, we assume that the received primary signal in PBS always has a desired $S N R=\frac{P_{p} G_{p p}}{L \sigma^{2}} \approx 10$. The capacity on each cluster occupied by $\mathrm{PU}$ is

$$
C=\log _{2}\left(1+\frac{P_{p} G_{p p}}{L \sigma^{2}}\right)
$$

where $P_{p}$ is the primary transmission power on one cluster, and $G_{p p}$ is the channel gain from PU to PBS. The value of $I_{t h}$ can be automatically generated by defining a tolerable capacity loss coefficient $\lambda$ according to

$$
(1-\lambda) C=\log _{2}\left(1+\frac{P_{p} G_{p p}}{L \sigma^{2}+I_{t h}}\right)
$$

The experimental results of the Perfectly Synchronized (PS) case between PUs and SUs are given for the sake of
TABLE I

SYSTEM SIMULATION PARAMETERS

\begin{tabular}{lcc}
\hline Parameter & Value & Unit \\
\hline Total bandwidth $B$ & 10 & $\mathrm{MHz}$ \\
Center frequency & 2.5 & $\mathrm{GHz}$ \\
Number of sub-carriers & 1024 & - \\
Number of sub-carriers per cluster $L$ & 18 & - \\
Power limit per subcarrier $P_{\text {sub }}$ & 5 & $\mathrm{mWatt}$ \\
Noise power per subcarrier & -134.10 & $\mathrm{dBm}$ \\
Channel delays & $10^{-9}[0,110,190,410]$ & $\mathrm{s}$ \\
Channel powers & {$[0,-9.7,-19.2,-22.8]$} & $\mathrm{dB}$ \\
Channel realizations & 200 & - \\
\hline
\end{tabular}

comparison with the results of OFDM and FBMC based CR networks. In addition, the performance of the case with perfect channel gain information is also investigated.

The averaged capacities at different interference levels $(\lambda=$ $0.02 \sim 0.2$ ) with a given outage probability $P_{\text {out }}=0.06$, a fixed maximum user power $P_{t h}=36 \mathrm{mWatt}$, and a fixed $D=0.2 \mathrm{~km}$ are given in Fig. 3. As expected, the performance of FBMC always outperforms that of OFDM, which shows a fast decrease of the channel capacity when less capacity loss is prescribed by PU, whereas FBMC is slightly affected by different interference levels. At the same time, we can see a large channel capacity gap between the case with ideal channel gain information and the case with an estimated channel gain for the OFDM based CR system, while there is a slight capacity difference by applying the FBMC based CR system. This could be explained by the fact that the number of subcarriers of OFDM and FBMC that induce harmful interference to PU are " 8 " and " 1 ", respectively. When a low outage probability is required, more subcarriers adjacent to PU should be deactivated or underutilized for OFDM, which accordingly degrades the amount of channel capacity. On the other hand, FBMC exhibits more distinct advantage than OFDM when a rough estimated channel gain is considered. Besides, it can be noted that the performance of FBMC approaches the performance of the perfectly synchronized case. Fig. 4 shows the averaged capacities versus different outage probabilities, where the averaged capacity (with an estimated channel gain) of OFDM based CR system collapses when a low outage probability is prescribed. On the contrary, FBMC based CR system is much less vulnerable to different outage probabilities.

The effect of the maximum power levels is illustrated in Fig. 5. It is seen that the channel capacities increase with the augmentation of the averaged power per subcarrier $\bar{P}$ $\left(\bar{P}=P_{t h} / \mathrm{F}\right)$. In view of the fact that the distance $D$ between SBS and PBS can be random because of the flexibility of cognitive radio, so the impact of $D$ on channel capacity is investigated and shown in Fig. 6. We can observe that as the distance increases, all the performance curves of FBMC and OFDM tend to merge. The reason is that there exists little interference between the primary system and the secondary cell when they are far away from each other. 


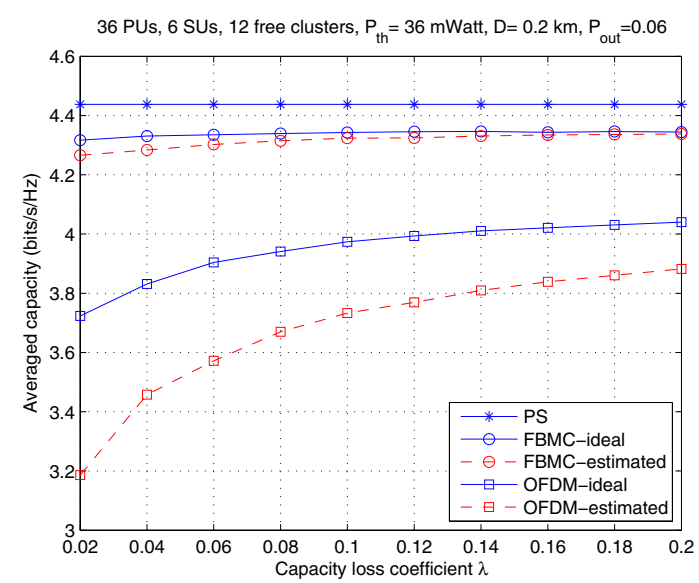

Fig. 3. Averaged capacity vs. interference level

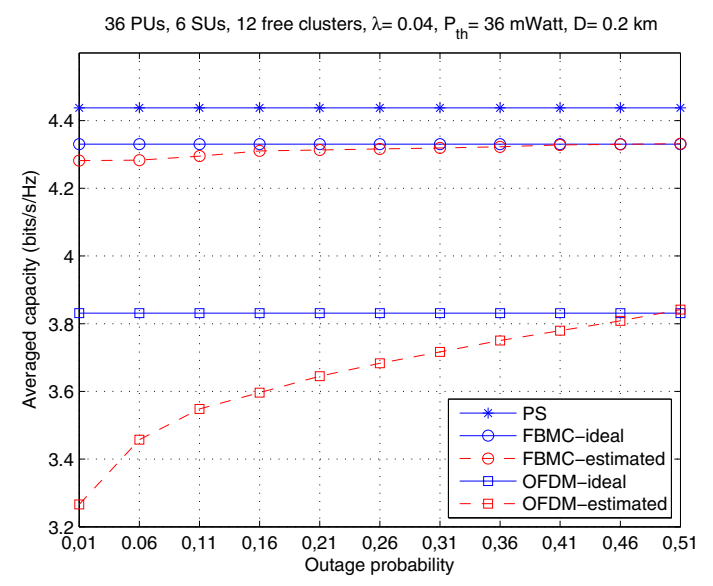

Fig. 4. Averaged capacity vs. outage probability

\section{Conclusion}

In this paper, a resource allocation algorithm with the considerations of power constraint and interference constraint is proposed for evaluating and comparing the averaged channel capacities of OFDM and FBMC based CR networks. Numerical results demonstrate that in our scenario FBMC can offer higher channel capacity and can achieve much more performance gain if rough estimated channel information is considered. Furthermore, the performance of FBMC is close to that of the perfectly synchronized case because of its frequency localization. As a result, we conclude that FBMC has practical value and is a potential candidate for physical layer data communication of future CR networks.

\section{REFERENCES}

[1] P. Siohan, C. Siclet, and N. Lacaille, "Analysis and design of OFDM / OQAM systems based on filterbank theory," IEEE Trans. Signal Processing, vol. 50, pp. 1170-1183, May 2002.

[2] M. Bellanger, "Filter banks and OFDM / OQAM for high throughput wireless LAN," in Proc. of 3rd International Symposium on Communications, Control and Signal Processing, Malta, Mar. 2008, pp. 758-761.

[3] B. Farhang-Boroujeny and R. Kempter, "Multicarrier Communication Techniques for Spectrum Sensing and Communication in Cognitive Radios," IEEE Communications Magazine, 46(4): 80-85, Apr. 2008.

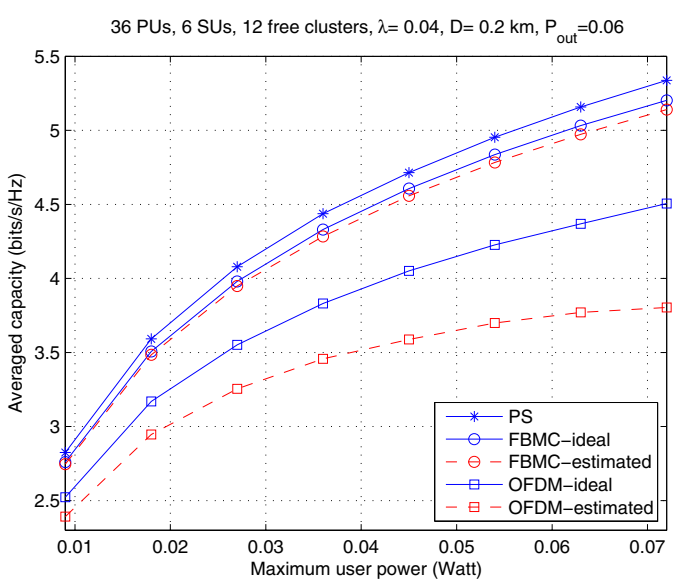

Fig. 5. Averaged capacity vs. maximum user power

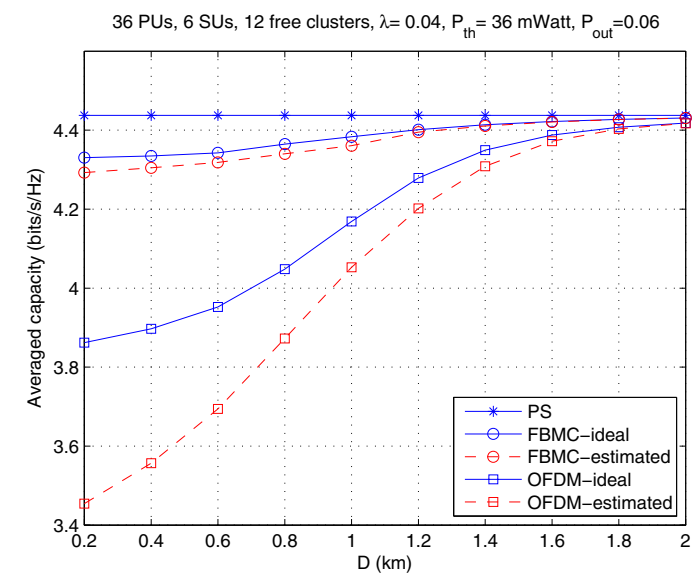

Fig. 6. Averaged capacity vs. distance between SBS and PBS

[4] H. Zhang, D. Le Ruyet, and M.Terré, "Spectral efficiency comparison between OFDM / OQAM and OFDM based CR networks," Wireless Communications and Mobile Computing, Wiley, vol. 9, pp. 1487-1501, Nov. 2008.

[5] B. Farhang-Boroujeny, "Filter Bank Spectrum Sensing for Cognitive Radios," IEEE Transactions on Signal Processing, vol. 56, iss. 5, pp. 1801-1811, May 2008.

[6] M. Shaat and F. Bader, "Computationally Efficient Power Allocation Algorithm in Multicarrier Based Cognitive Radio," accepted to EURASIP Journal on Advances in Signal Processing.

[7] H. Zhang, D. Le Ruyet, D. Roviras, Y. Medjahdi, and H. Sun, "Spectral Efficiency Comparison of OFDM / FBMC for Uplink Cognitive Radio Networks," accepted to EURASIP Journal on Advances in Signal Processing.

[8] Y. Medjahdi, M. Terré, D. Le Ruyet, D. Roviras, J. A. Nossek, and L. Baltar, "Inter-cell Interference Analysis for OFDM / FBMC Systems," in Proc. of 10th IEEE Signal Processing Workshop (SPAWC 2009), Perugia, Italy, Jun. 2009, pp. 598-602.

[9] M. S. Barzaraa, H. D. Sherali, and C. M. Shetty, "Nonlinear programming: theory and algorithms," 2ed ed, John Wiley \& Sons, 1993.

[10] C. Lengoumbi, P. Godlewski, and P. Martins, "An Efficient Subcarrier Assignment Algorithm for Downlink OFDMA," in Proc. of Vehicular Technology Conference, Sep. 2006, pp. 1-5.

[11] H. W. Kuhn, "Variants of the Hungarian method for assignment problems," Naval Research Logistics Quarterly, 3: 253-258, 1956.

[12] Draft IEEE $802.16 \mathrm{~m}$ Evaluation Methodology Document C80216m-07080r2. 